OPEN ACCESS

Edited by:

Miloslav Pekar̆

Brno University of Technology,

Czechia

Reviewed by:

Anna Trybala,

Loughborough University, United Kingdom

Hock Jin Quah

University of Science Malaysia,

Malaysia

*Correspondence:

Dianqiao Geng

gengdianqiao@epm.neu.edu.cn

Specialty section:

This article was submitted to Colloidal Materials and Interfaces,

a section of the journal

Frontiers in Materials

Received: 11 May 2021

Accepted: 30 July 2021

Published: 11 August 2021

Citation:

Geng D, Li J, Li H and Huang W (2021) Effects of Particle Combinations With Different Wettability on Foam Structure and Stability.

Front. Mater. 8:699187. doi: 10.3389/fmats.2021.699187

\section{Effects of Particle Combinations With Different Wettability on Foam Structure and Stability}

\author{
Dianqiao Geng ${ }^{1,2 *}$, Jiahui $L i^{1}$, Huaying $L i^{3}$ and Wenzhan Huang ${ }^{3}$ \\ ${ }^{1}$ Key Laboratory of Electromagnetic Processing of Materials, Ministry of Education, Northeastern University, Shenyang, China, \\ ${ }^{2}$ School of Metallurgy, Northeastern University, Shenyang, China, ${ }^{3}$ School of Materials Science and Engineering, Taiyuan \\ University of Science and Technology, Taiyuan, China
}

Particle addition is an important method to prepare foam metal, and it is of great significance to clarify the mechanism of particle stabilizing liquid metal foam. In this paper, ethanol-water solution system is used to simulate liquid melt foam. By changing the wettability of particles to adjust the distribution position of particles in foam, two types of particles with different wettability are added, which are mixed and optimized in a certain proportion to improve the foam stability as much as possible. The main mechanism is that the large wetting angle particles at the gas-liquid interface to slow down the gas migration, while small wetting angle particles exist in the liquid film, which can reduce the liquid drainage velocity. The experimental results show that the effect of particle wettability on foam structure is greater than that on viscosity enhancement. The particles with large wetting angle are beneficial to the formation and stability of foam, and the particles with small wetting angle cannot stabilize the foam alone. The effect of two types of particle combinations with different wettability on foam stability is better than that of single type of particle. Considering the height and uniformity of the foam structure, the optimal particle combination is finally obtained.

Keywords: metal foam, particle, combination, wettability, stability

\section{INTRODUCTION}

As one of the most potential new functional materials, closed-cell metal foam has a wide application prospect in various fields such as military industry, fire protection and aerospace because of its characteristics of light mass, large specific strength, and large specific stiffness (Banhart, 2013; Liu et al., 2013; Xu and Hao, 2014). For example, the foam sandwich plate with foam aluminum as the intermediate core layer has been used for trains and other vehicles ( $\mathrm{Zu}$ et al., 2011), which can not only reduce the weight of the vehicles and save energy, but also can be used as a collision absorber to improve safety performance (Banhart, 2005; Garcia-Moreno, 2016). Moreover, with small thermal conductivity and light quality (Wang et al., 2016; Li et al., 2020), the closed-cell metal foam is expected to become a new insulation material (Zhu et al., 2004).

In foam metal preparation process, particles or other elements are usually added to improve the stability of foam structure. For example, $\mathrm{Ca}$ is added to the melt and stirred in the ambient atmosphere to form the oxide inside metal melts to achieve foam stability, which is also called thickening (Miyoshi et al., 2000; Song et al., 2000; Heim et al., 2017; Cheng et al., 2018). Moreover, Fe or Si has been added in the basic alloy AlSi9Mg0.6 in the first stage of solidification of eutectic alloys to foam Primary Fe and Si-rich crystals which can improve the foam stability (Heim et al., 2018). Furthermore, Mg has also been used as thickener 
to stabilize the foams (Heim et al., 2015). And some studies have shown that $\mathrm{Mg}$ addition can results in the formation of $\mathrm{MgAl}_{2} \mathrm{O}_{4}$ particles which stabilize the foams (Bhogi and Mukherjee, 2017). Such compounds can inhibit the liquid drainage process inside the liquid film. Besides, ceramic particles such as SiC (Wang and Shi, 2003; Elbir et al., 2003; Afshar et al., 2017; Kumar and Gokhale, 2018) are added to directly increase viscosity, thus increasing the stability of foam structure and improving the uniformity of cell pores. However, the effect of the particle on the stability of foam structure and its mechanism are not the same. Hosseini et al. (2016) found that most of the $\mathrm{SiC}$ particles were distributed around the foaming agent particles, thus improving the foam structure. Kumar et al. (2014) observed that the SiC particles did not gather in large quantities at the interface, but were distributed inside the cell wall, presumably stabilizing the foam by increasing viscosity. However, the information about wettability of $\mathrm{SiC}$ in literatures is insufficient. It is possible that the pretreatment of particles in the experimental process is different, resulting in different wetting angles, which affects the different distribution positions of $\mathrm{SiC}$ particles in metal foam. Beside $\mathrm{SiC}$ particles, $\mathrm{Al}_{2} \mathrm{O}_{3}$ or $\mathrm{SiO}_{2}$ particles have been added in the melt by stirring method (Leitlmeier et al., 2002; Babcsan et al., 2004; Garcia-Moreno et al., 2005). On the other hand, it should be pointed out that the above methods of adding different elements also stabilize the foam by generating particles.

However, there are different explanations for the mechanism of particle stabilized metal foam. Liu et al. (2011) found that the wettability of particles has a greater effect on foam stability than the viscosity enhancement. Some researchers have also studied the relationship between particle wetting angle and foam stability. Kaptay (2003) believed that the optimum wetting angle range of ceramic particle stabilized metal foam is $70^{\circ}-86^{\circ}$. Sun and Gao (2002) believed that the optimum wetting angle range is $75^{\circ}-85^{\circ}$, and the foam has a better cell structure in this range. Also another study shows that the optimal wetting angle is $90^{\circ}$ for foam stability (Kaptay, 2012).

It is difficult to adjust the wettability of ceramic particles quantitatively during the preparation of foam metal. On the other hand, the distribution position of particles in foam can be regulated by changing the wettability of particles in ethanolwater solution. Therefore, the main purpose of this paper is to stabilize the foam by adding two types of particles with different wetting angle, and to maximize the role of stabilization foam by changing different proportions. The wetting angle of particles is adjusted by changing the solution composition. It is expected that this work will provide a new insight for the synergistic preparation of foam metals based on different wettability of two particles. The paper is organized as follows: Experiment introduces experiment and explains in detail. In Effect of Wettability of Single Type Particles-Effect of Size of Single Type Particles, the effects of wettability, concentration and size of single type particles are presented and analyzed in detail to quantify the range of particle parameters. Effect of Particle Combinations With Different Wettability shows the effect of particle combinations with different wettability on foam structure and stability. In Optimization of Particle Combinations With Different Wettability, the particle combinations with different wettability are optimized based on the foam structure and foam height. Finally, the conclusions are drawn in Conclusion.
TABLE 1 | Parameters of particles in experiments.

\begin{tabular}{|c|c|c|c|c|c|}
\hline \multirow[b]{2}{*}{ Particle size $(\mu \mathrm{m})$} & \multicolumn{4}{|c|}{ PTFE particle } & \multirow{2}{*}{$\begin{array}{c}\text { PVC particle } \\
270\end{array}$} \\
\hline & 0.5 & 5 & 20 & 48 & \\
\hline Particle density $\left(\mathrm{g} / \mathrm{cm}^{3}\right)$ & 2.2 & 2.2 & 2.2 & 2.2 & 1.3 \\
\hline
\end{tabular}

\section{EXPERIMENT}

An ethanol-water system was used to simulate the metal melt foam. Water and ethanol can form a continuous insoluble solution, which is similar to molten metal. By changing the composition of ethanol-water solution, the wetting angle of particles can be controlled to analyze the influence of particle wetting properties on the foam structure and stability. On the other hand, the PTFE and PVC particles, similar to $\mathrm{SiC}$ in $\mathrm{Al}$, are inert in the ethanol-water solution. Furthermore, the wettability of PTFE and PVC particles is also different under the same solution and decreases with the increasing volume fraction of ethanol. Therefore, the PTFE and PVC particles (Dongguan, China, Xingwang Co., Ltd.) with different size are used to simulate the effects of ceramic particles with different wetting properties on the foam structure and stability. In addition, no surfactant was added. The particle parameters are shown in Table $\mathbf{1}$.

The foam was prepared by the same method as literature (Sun and Gao, 2002). The main steps are as follows. The corresponding ethanol-water solution was used to clean the test tube. Then the $10 \mathrm{~g}$ of ethanol-water solution and particles were added into the test tube. The test plug was pressed to shake until the foam volume no longer increases. After generating a certain height of foam, the test tube is perpendicular to the horizontal plane. Photos were taken after 2 and $10 \mathrm{~min}$, respectively. Each group of experiments was repeated five times to avoid experimental contingency. It should be pointed out that the foam height prepared under the same conditions is basically the same during the experiment.

The photos were imported into software Image-Pro Plus 6.0. The foam profile was determined based on the processed image. Then the average size of foam cell can be obtained. The viscosity of the suspension after adding particles was measured by rotary viscometer (NDJ-9S, Shanghai, China, Pingxuan Scientific Instrument Co., Ltd.), and the corresponding viscosity value was recorded after stirring. The contact angle meter (JC300DI, Sweden, Biolin Scientific Co., Ltd.) was used to measure the corresponding wetting angle by the drop method. Besides, the optical microscopy (PH50, Jiangxi, China, Phoenix Optical Instrument Co., Ltd.) was used to observe and photograph the microstructure of foam.

\section{RESULTS AND DISCUSSION}

\section{Effect of Wettability of Single Type Particles}

The foam was prepared with PTFE particles of $20 \mu \mathrm{m}$ size, and the mass fraction was $1.0 \mathrm{wt} \%$. By changing the ethanol concentration to adjust the wetting angle of the particles, the effect of different wetting angles on the stability of the foam was analyzed.

Figure 1 shows the photo of foam at different ethanol concentrations after $10 \mathrm{~min}$. The foam structure remains intact 


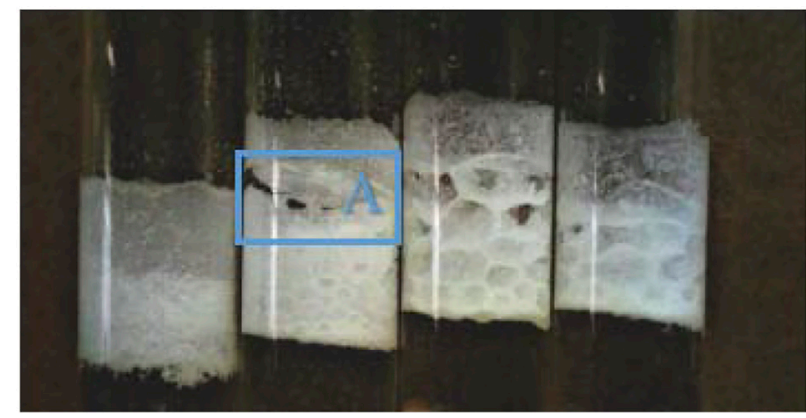

FIGURE 1 | Foam structure with different ethanol concentrations (From left to right: 20.0 vol\%, 30.0 vol\%, 40.0 vol\%, and 50.0 vol\% ethanol; PTFE, $20 \mu \mathrm{m}, 1.0 \mathrm{wt} \%)$.

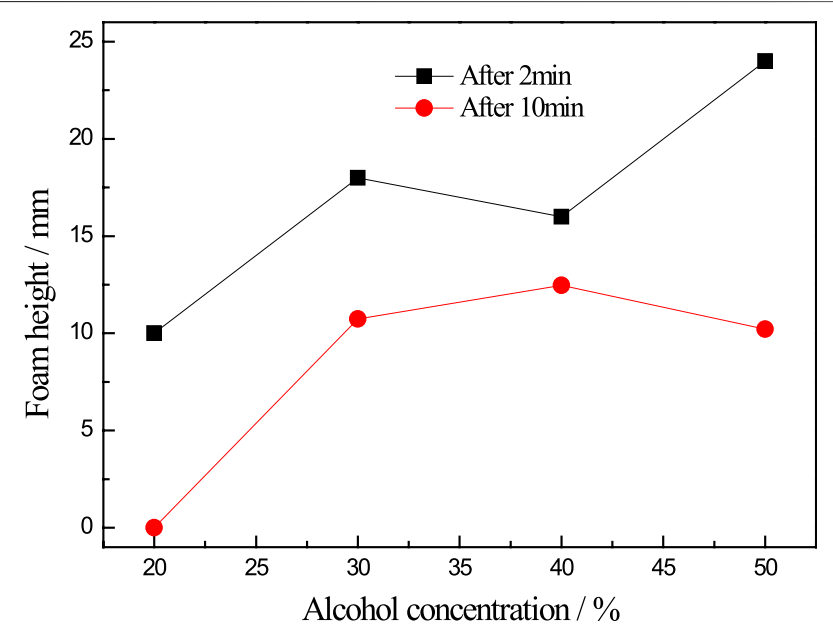

FIGURE 2 | Foam height with different ethanol concentrations (PTFE, $20 \mu \mathrm{m}, 1.0 \mathrm{wt} \%)$.

when the ethanol concentration is $40.0 \mathrm{vol} \%$. The particles in the solution agglomerate to form flocculent, and there is no foam structure when the ethanol concentration is 20.0 vol\%. Moreover, the cells in the upper part of the foam merge and burst when the ethanol concentration is $30.0 \mathrm{vol} \%$ or $50.0 \mathrm{vol} \%$, which can be found in Figure 1 (Part A).

Figure 2 shows that the difference of foam height after 2 and $10 \mathrm{~min}$ is the least when the ethanol concentration is $40.0 \mathrm{vol} \%$. Therefore, the corresponding wetting angle is the best value, which is consistent with the experimental results in the literature (Sun and Gao, 2002). Moreover, When the ethanol concentration is $20.0 \mathrm{vol}$ $\%$, the foam lifetime is between 2 and $10 \mathrm{~min}$ because the foam disappears after $10 \mathrm{~min}$. When the ethanol concentration is between 30 and $50 \%$, the corresponding foam lifetime exceeds $10 \mathrm{~min}$.

\section{Effect of Concentration of Single Type Particles}

Figure 3 shows the structure of foams adding different particle concentration when the ethanol concentration is $40 \%$. The foam

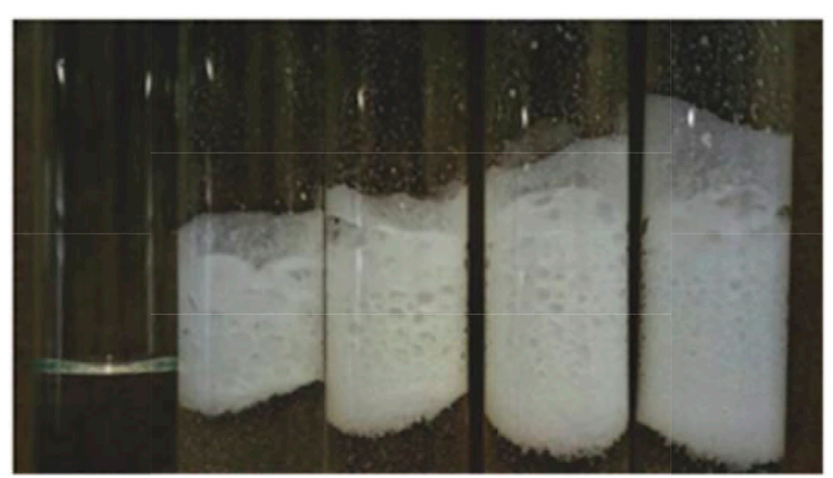

FIGURE 3 | Foam structure with different particle concentrations (From left to right: 0 wt\% PTFE, 1.0 wt\% PTFE, 2.0 wt\% PTFE, 3.0 wt\% PTFE,

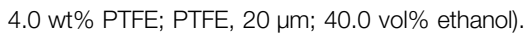

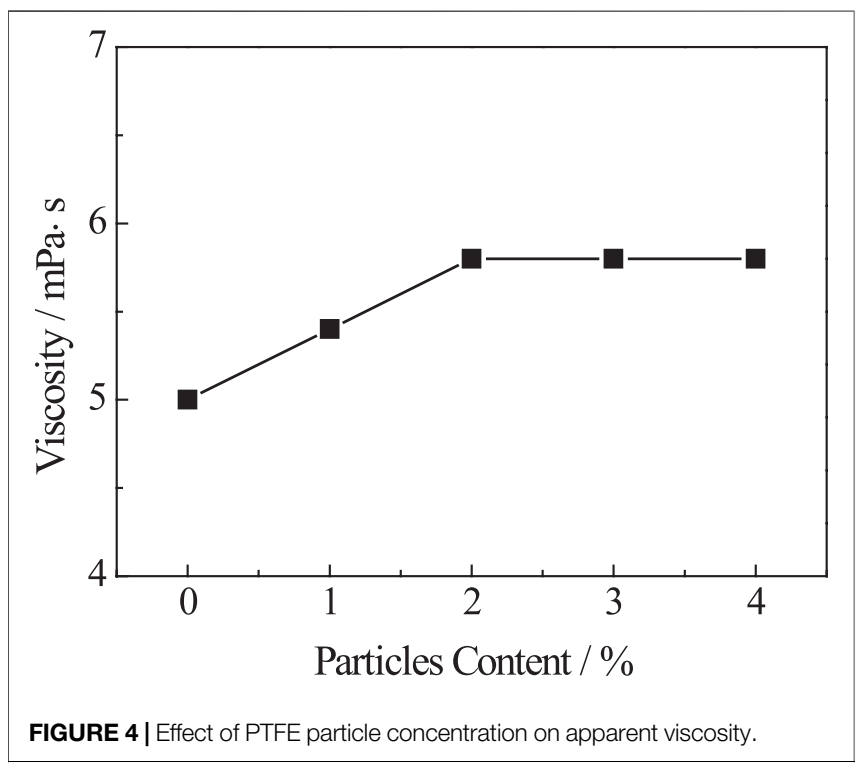

is not generated without adding particles. The foam height increases with the increasing particle concentration, and the size of foam structure becomes obviously smaller, which is consistent with the experimental results in the literature (Lin et al., 2017). When the particle concentration increases to $4.0 \mathrm{wt}$ $\%$, due to the poor wettability, the particles accumulates inside the foam structure after the foam formation, which leads to dense layer at the foam bottom.

Figure 4 shows that the apparent viscosity increases from $5.0 \mathrm{mPa}$ s to $5.8 \mathrm{mPa}$, when the particle concentration increases to $2.0 \mathrm{wt} \%$. And the apparent viscosity is almost unchanged when the content changes from $2.0 \mathrm{wt} \%$ to $4.0 \mathrm{wt} \%$. Thus, there are other mechanisms to stabilize the foam in addition to viscosity enhancement.

Figure 5 shows the plateau boundary formed by three bubbles. Since the wetting angle of PTFE particles is $76^{\circ}$, PTFE particles are adsorbed at the gas-liquid interface in 


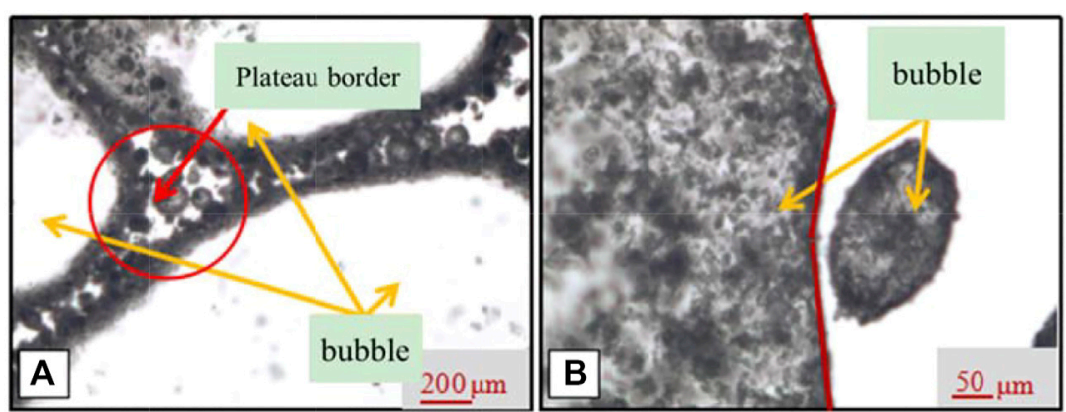

FIGURE 5 | Microstructure of foam. (A) Plateau border (B) Partial enlarged picture.

TABLE 2 | Parameters of particles in experiments.

\begin{tabular}{lcccc}
\hline & \multicolumn{3}{c}{ PTFE particle } & PVC particle \\
\hline Particle concentration $(w t \%)$ & 2.0 & 2.0 & 2.0 & 1.0 \\
Particle size $(\mu \mathrm{m})$ & 0.5 & 5 & 48 & 270
\end{tabular}

the foam structure, and there are almost no particles in the liquid film. Therefore, the main mechanism of the PTFE particles with poor wettability in the foam structure is the influence of the distribution position, rather than the viscosity enhancement.

\section{Effect of Size of Single Type Particles}

In order to analyze the effect of particle size on foam stability, the experiment is carried out as shown in Table 2. At the same time, a group of high wettability particles were added for comparison.

The PTFE particles of size $48 \mu \mathrm{mm}, 5$, and $0.5 \mu \mathrm{m}$ were added under the condition of ethanol concentration of $40.0 \mathrm{vol} \%$ and particle concentration of $2.0 \mathrm{wt} \%$, respectively. The experimental results are as follows. The particles formed flocculent in the process of tube oscillation, and no foam structure was formed when the particle size is $48 \mu \mathrm{m}$. The bubble quickly disappears and cannot form foam when the particle size is 5 and $0.5 \mu \mathrm{m}$.

As shown in Figure 6, the blue circle represents the particle. The dark gray area represents the bubble. The light gray area represents the liquid film. The yellow line represents the gas- liquid interface. And the arrow represents the direction of liquid evolution.

Figure 6B shows that the particles are close to the liquid side when the wetting angle of the particles is small, so it is easy to separate from the gas-liquid interface. If the viscosity enhancement effect is insufficient, the particles are easy to be taken away during the drainage process. Figure 6C shows that the particles are easily taken away during the liquid drainage process when the particle size is too small, and finally accumulate at the bottom of the foam. Therefore, as shown in Figure 6A, when the wettability of particles is poor and the size is moderate, it is an ideal situation. Moreover, our experimental results also show that the foam disappears quickly after foam formation for PVC particles with high wettability, which proves that foam stability is related to the wettability of particles. The main mechanism is that the wettability of particles determines the relative position between particles and gas-liquid interface.

\section{Effect of Particle Combinations With Different Wettability}

According to the above analysis, the particles with large wetting angle are distributed at the gas-liquid interface, which plays a decisive role in stabilizing the foam structure, and the particles with small wetting angle are distributed inside the liquid, which can stabilize the foam structure by increasing viscosity and suppress the drainage behavior. Therefore, the

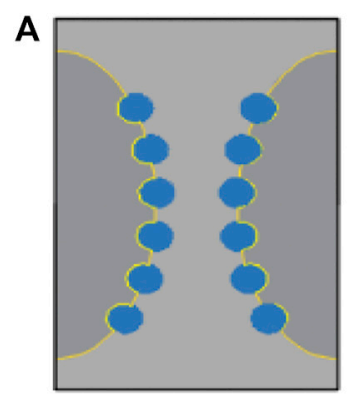

B

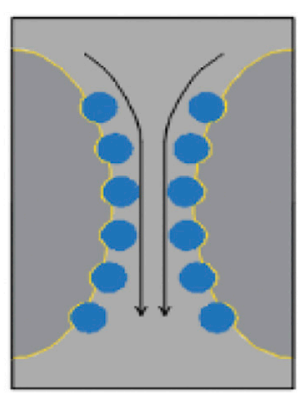

C

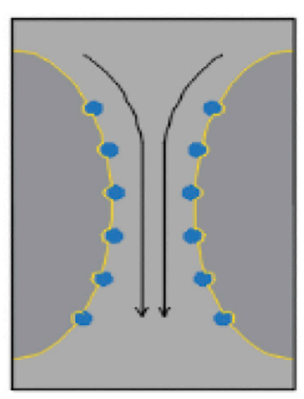

FIGURE 6 | Position distribution of particles and bubbles in foam. (A) Particles with poor wettability (B) Particles with good wettability (C) Too small particles. 


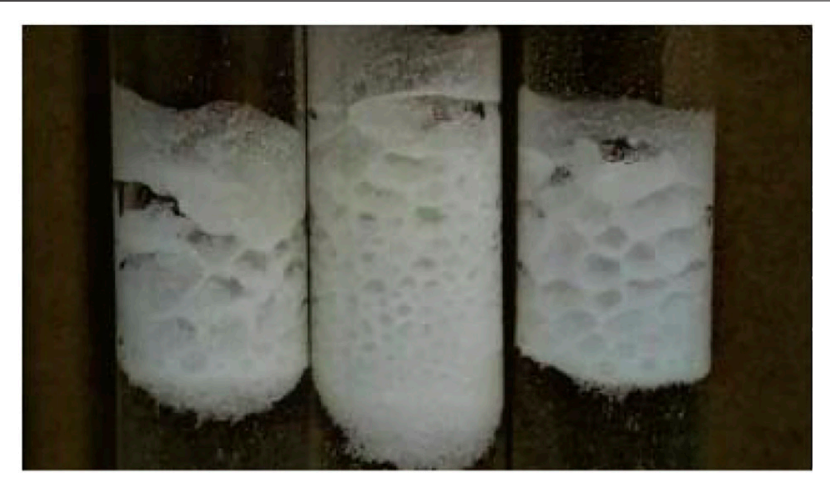

FIGURE 7 | Foam structure with different particle combinations (From left to right: 1.0 wt\% PTFE, 2.0 wt\% PTFE, 1.0 wt\% PTFE, and 1.0 wt\% PVC; PTFE, $20 \mu \mathrm{m}, \mathrm{PVC}, 270 \mu \mathrm{m})$.

TABLE 3 | Group of particle combinations.

\begin{tabular}{lccccccc} 
Group & PTFE concentration (wt\%) & \multicolumn{5}{c}{ PVC concentration (wt\%) } \\
\hline A & 1.5 & 0.3 & 0.6 & 0.9 & 1.2 & 1.5 & 1.8 \\
B & 2 & 0.3 & 0.6 & 0.9 & 1.2 & 1.5 & 1.8 \\
C & 2.5 & 0.3 & 0.6 & 0.9 & 1.2 & 1.5 & 1.8 \\
D & 3 & 0.3 & 0.6 & 0.9 & 1.2 & 1.5 & 1.8
\end{tabular}

effect of particles in the foam structure is different with the different wetting angles. It can be speculated that adding large wetting angle particles to form foam. Moreover, adding small wetting angle particles can inhibit the drainage, and further stabilizing the foam structure. That is, two particles with different wettability can work together to optimize the foam structure.

Figure 7 shows that there is almost no dense layer at the bottom of the foam formed by mixing two types of particles compared with single type of particles, which indicates that the combination of the two types of particles has a better suppression effect on drainage behavior. At the same time, the foam structure is obviously better than that of single type of particles. The reason is that the wettability of PVC particles is good on the condition of $40.0 \mathrm{vol} \%$ ethanol concentration and can be distributed within the liquid film. The foam cannot be generated when PVC particles are added separately. However, after mixing with PTFE particles, foam can be formed and stabilized. It shows that two types of particle combinations with different wettability can effectively improve the foam stability.

As shown in Table 3, according to the PTFE particle concentration, four groups were divided. The optimal particle concentration combination can be obtained by comparing the foam structure under different particle combinations.

Figure 8 shows the foam structure with different particle combinations. The white area represents the overall height of the foam. Irregular color regions represent different cells of foam. The white area at the bottom of the foam represents the dense layer of particles. The cell size decreases gradually from top to bottom because of the bubble coalescence. With the increasing PTFE particle concentration, the PTFE particles at

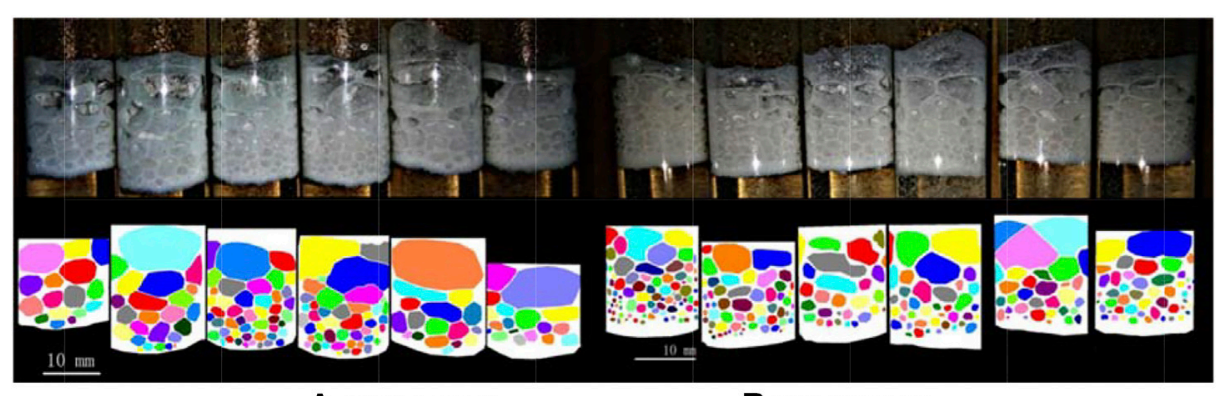

A PTFE 1.5 wt \%

B PTFE $2.0 \mathrm{wt} \%$

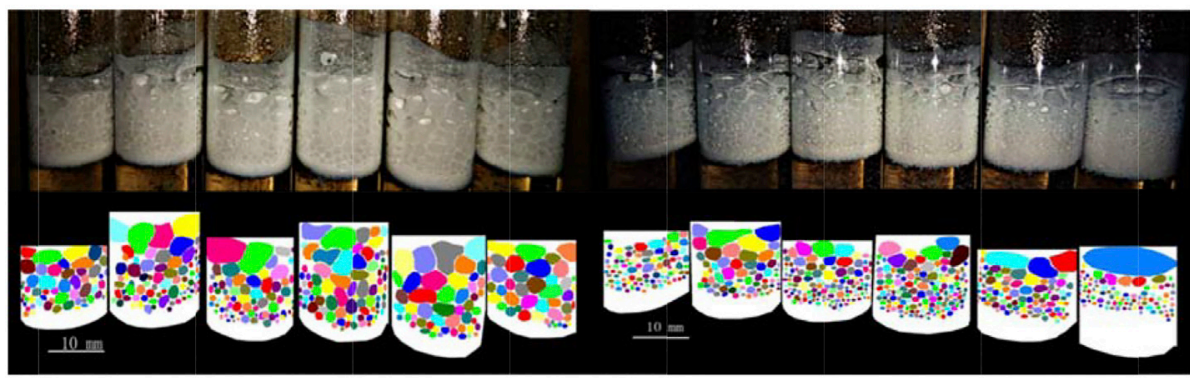

C PTFE 2.5wt \%

D PTFE $3.0 \mathrm{wt} \%$

FIGURE 8 | Foam structure with different PVC particle concentration (Each graph from left to right: 0.3 wt $\%, 0.6 w t \%, 0.9$ wt $\%, 1.2$ wt $\%, 1.5$ wt $\%$, and 1.8 wt $\%$ PVC). (A) PTFE 1.5 wt\% (B) PTFE 2.0 wt $\%$ (C) PTFE 2.5 wt $\%$ (D) PTFE 3.0 wt\%. 


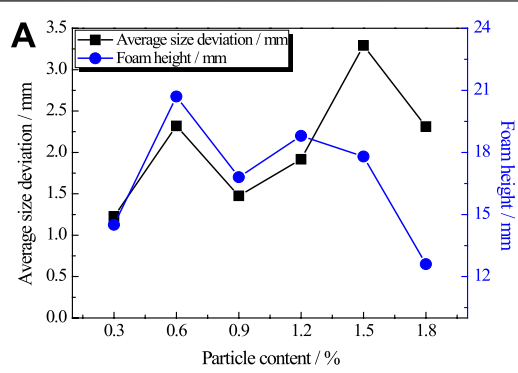

PTFE $1.5 \mathrm{wt} \%$

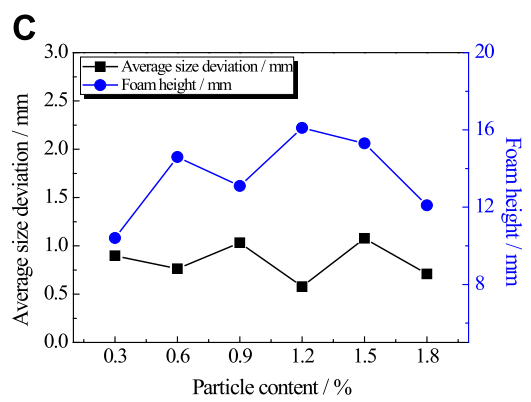

PTFE $2.5 w t \%$

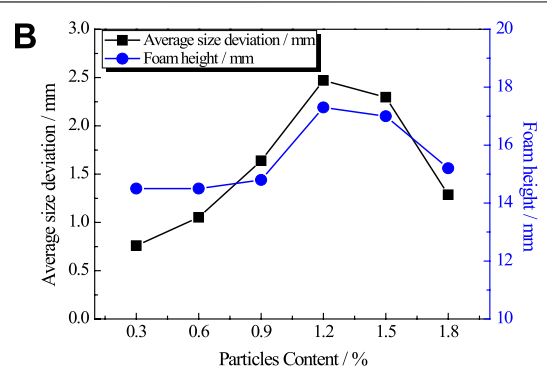

PTFE 2.0wt \%

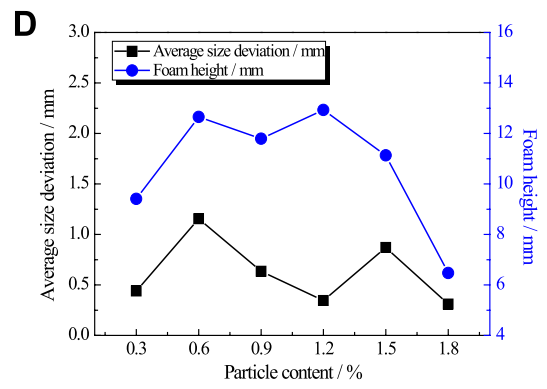

PTFE 3.0wt \%

FIGURE 9 | Foam height and average cell size deviation under different PVC particle concentrations. (A) PTFE 1.5 wt\% (B) PTFE 2.0 wt\% (C) PTFE 2.5 wt\% (D) PTFE 3.0 wt\%.

TABLE 4 | Results of optimal particle combination.

Group

PTFE concentration (wt\%)

PVC concentration (wt\%)

a (A group)

b (B group)

c (C group)

d (D group)

1.5
2.0
2.5
3.0

1.5

0.6

1.2

3.0

the gas-liquid interface increase and the stability of the foam is enhanced, so the cell size of the group B becomes smaller and the large bubble in the upper part of the foam disappears, while the cell shape of group C and D is approximately circular. At the same time, with the increasing PTFE particle concentration, the thickness of dense layer at the bottom of foam increases gradually.

In order to evaluate the uniformity of foam structure, the foam is divided into two regions along the height direction, and then the average size of bubbles in the two regions is measured, respectively.

Figure 9 shows that the curve of average size deviation changes with the increasing PVC particle concentration under different PTFE particle concentration conditions. When the concentration of PVC particles is low, the bubbles are easy to merge, which leads to the increase of foam height, but the overall uniformity of foam becomes worse. As the concentration of PVC particles increases further, the effect of suppression of drainage behavior increases, and there is no bubble merger at the top of the foam. Therefore, the foam height decreases and the overall

uniformity of the foam become better. After that, the PVC particles aggregate, and the liquid film widens, which accelerates the drainage. Then the upper part of the foam merges to form a large bubble and collapses, causing a reduced foam height and a dense layer at the bottom of the foam.

\section{Optimization of Particle Combinations With Different Wettability}

When evaluating the optimal particle combination of each group, the uniformity of cell size is taken as the main standard, followed by foam height. And the optimal particle combinations of each group are shown in Table 4.

Figure 10 shows the cell size distribution under optimal particle combinations in Table 4. $\mathrm{D}_{\text {mean }}$ is the average diameter of the foam cell after Gaussian normal fitting. With the increasing PTFE particle concentration, the average size of foam cell decreases and the size distribution is more concentrated. The average size of the group $d$ reached the minimum, and the size distribution was the most concentrated, mainly in the range of $0.4-1.0 \mathrm{~mm}$, accounting for $68 \%$ of the total.

Figure 11 shows the corresponding foam height of four optimal particle combinations. The foam height is random because the uniformity of foam structure is taken as the main factor. If only the uniformity of the foam structure is considered, the particle combination of the group $d$ is the best. Furthermore, if the uniformity of the foam structure and the foam height are considered simultaneously, the particle content of the $c$ group is optimal. 

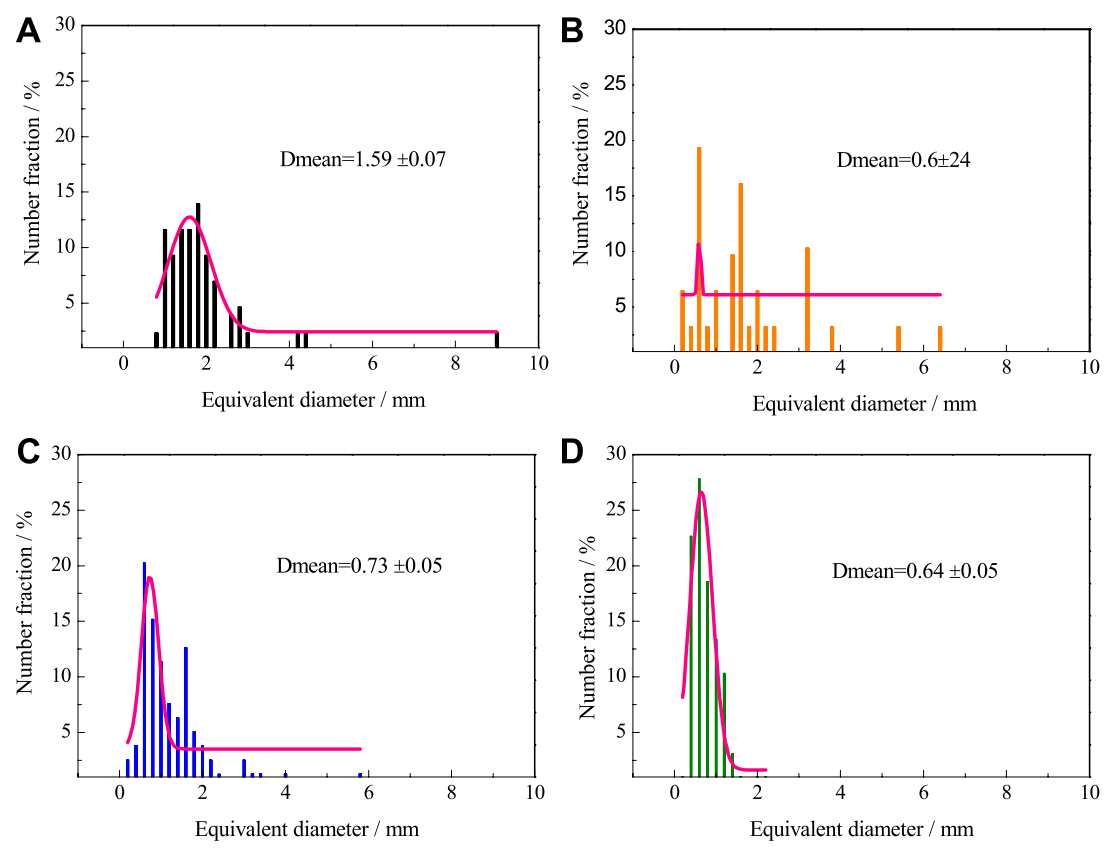

FIGURE 10 | Distribution of foam cell size in Table 4.

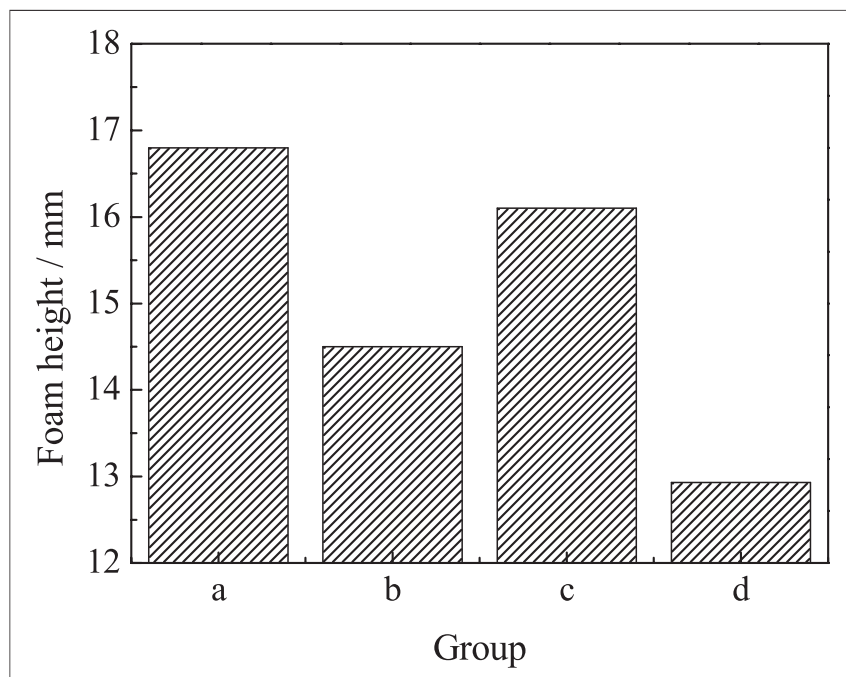

FIGURE 11 | Foam height with different particle combination after 2 min.

\section{CONCLUSION}

The ethanol solution is used to simulate the formation of metal foam. Two types of particles with different wettability were grouped to compare the corresponding foam height and foam structure. The effect of particle wettability on foam structure is greater than that of viscosity enhancement. The particles with large wetting angle are beneficial to the formation and stability of foam, and the particles with small wetting angle cannot stabilize the foam alone. The effect of two types of particle combinations with different wettability on foam stability is better than that of single type particles. The main mechanism is that the particles with large wetting angle exist at the gas-liquid interface to slow the gas migration, while the particles with small wetting angle exist in the liquid film and can reduce the liquid drainage velocity. Considering the foam height and foam structure, the optimal particle combination is obtained.

\section{DATA AVAILABILITY STATEMENT}

The original contributions presented in the study are included in the article/supplementary material; further inquiries can be directed to the corresponding author.

\section{AUTHOR CONTRIBUTIONS}

DG contributed to the conception of the study and manuscript preparation; JL performed the experiment and the data analyses; $\mathrm{HL}$ and WH helped perform the analysis with constructive discussions of the manuscript.

\section{FUNDING}

This work was supported by the open funds of Key Laboratory of Electromagnetic Processing of Materials, Ministry of Education, Northeastern University (NEU-EPM-014), the National Natural Science Foundation of China (51304038) and the Fundamental Research Funds for the Central Universities (N180904007). 


\section{REFERENCES}

Afshar, M., Mirbagheri, M. H., and Movahedi, N. (2017). Effect of SiC Particle Size on the Mechanical Properties of Closed Aluminum Foams. Mater. Test. 59 (6), 571-574. doi:10.3139/120.111035

Babcsán, N., Leitlmeier, D., Degischer, H. P., and Banhart, J. (2004). The Role of Oxidation in Blowing Particle-Stabilised Aluminium Foams. Adv. Eng. Mater. 6 (6), 421-428. doi:10.1002/adem.200405144

Banhart, J. (2005). Aluminium Foams for Lighter Vehicles. Ijvd 37 (2-3), 114-125. doi:10.1504/ijvd.2005.006640

Banhart, J. (2013). Light-Metal Foams-History of Innovation and Technological Challenges. Adv. Eng. Mater. 15 (3), 82-111. doi:10.1002/adem.201200217

Bhogi, S., and Mukherjee, M. (2017). Foam Stabilization by Magnesium. Mater. Lett. 200, 118-120. doi:10.1016/j.matlet.2017.04.100

Cheng, Y., Li, Y., Chen, X., Liu, Z., Zhou, X., and Wang, N. (2018). Optimizing Calcium Addition for Fabricating Aluminum Foams with Different Pore Sizes. Mater. Trans. 59 (8), 1367-1374. doi:10.2320/matertrans.M2018131

Elbir, S., Yilmaz, S., Toksoy, A. K., and Guden, M. (2003). SiC-particulate Aluminum Composite Foams Produced by Powder Compacts: Foaming and Compression Behavior. J. Mater. Sci. 38 (23), 4745-4755. doi:10.1023/A:1027427102837

Garcia-Moreno, F., Babcsan, N., and Banhart, J. (2005). X-ray Radioscopy of Liquid Metalfoams: Influence of Heating Profile, Atmosphere and Pressure. Colloids Surf. a-Physicochemical Eng. Aspects 263 (1-3), 290-294. doi:10.1016/ j.colsurfa.2004.12.044

Garcia-Moreno, F. (2016). Commercial Applications of Metal Foams: Their Properties and Production. Materials 9 (2), 85. doi:10.3390/ma9020085

Heim, K., García-Moreno, F., and Banhart, J. (2018). Particle Size and Fraction Required to Stabilise Aluminium alloy Foams Created by Gas Injection. Scripta Materialia 153, 54-58. doi:10.1016/j.scriptamat.2018.04.041

Heim, K., Vinod-Kumar, G. S., García-Moreno, F., and Banhart, J. (2017). Stability of Various Particle-Stabilised Aluminium Alloys Foams Made by Gas Injection. J. Mater. Sci. 52 (11), 6401-6414. doi:10.1007/s10853-017-0874-3

Heim, K., Vinod-Kumar, G. S., Garcia-Moreno, F., Rack, A., and Banhart, J. (2015). Stabilisation of Aluminium Foams and Films by the Joint Action of Dispersed Particles and Oxide Films. Acta Materialia 99, 313-324. doi:10.1016/j.actamat.2015.07.064

Hosseini, S. M., Habibolahzadeh, A., Petráňová, V., and Něme?Ek, J. (2016). Influence of Nano-SiCp on the Foamability and Microstructure of $\mathrm{Al} / \mathrm{TiH} 2$ Foam Sheet Manufactured by Continual Annealing and Roll-Bonding Process. Mater. Des. 97 (5), 483-491. doi:10.1016/j.matdes.2016.02.106

Kaptay, G. (2003). Interfacial Criteria for Stabilization of Liquid Foams by Solid Particles. Colloids Surf. a-Physicochemical Eng. Aspects 230 (1-3), 67-80. doi:10.1016/j.colsurfa.2003.09.016

Kaptay, G. (2012). On the Optimum Contact Angle of Stability of Foams by Particles. Adv. Colloid Interf. Sci. 170 (1-2), 87-88. doi:10.1016/j.cis.2011.12.002

Kumar, N. V. R., and Gokhale, A. A. (2018). Role of Temperature and SiCP Parameters in Stability and Quality of Al-Si-Mg/SiC Foams. JOM 70 (6), 823-828. doi:10.1007/s11837-018-2825-0

Kumar, N. V. R., Rao, N. R., and Gokhale, A. A. (2014). Effect of SiC Particle Content on Foaming and Mechanical Properties of Remelted and Diluted A356/SiC Composite. Mater. Sci. Eng. A 598 (26), 343-349. doi:10.1016/ j.msea.2014.01.050

Leitlmeier, D., Degischer, H. P., and Flankl, H. J. (2002). Development of a Foaming Process for Particulate Reinforced Aluminum Melts. Adv. Eng. Mater. 4 (10), 735-740. doi:10.1002/1527-2648(20021014)4:10<735::Aid-adem735>3.0.Co;2-y

Li, J., Geng, D., Zhang, R., Huo, X., and Wang, P. (2020). Effective Thermal Conductivity Modeling of Closed-Cell Aluminum Foam and Heat Transfer
Simulation. Rare Metal Mater. Eng. 49 (5), 1637-1642. 10/CNKI:SUN: COSE.0.2020-05-024

Lin, H., Luo, H., Zhang, Z., Ma, J., and Yao, G. (2017). Study on Improvement of Aluminum Foams by Rapid Foaming. Mater. Lett. 188 (1), 288-290. doi:10.1016/j.matlet.2016.10.109

Liu, H., Cao, Z. K., Luo, H. J., Shi, J. C., and Yao, G. C. (2013). Performance of Closed-Cell Aluminum Foams Subjected to Impact Loading. Mater. Sci. Eng. A 570, 27-31. doi:10.1016/j.msea.2012.11.094

Liu, X. N., Li, Y. X., Chen, X., and Fan, X. L. (2011). Effect of Ca on Particulate Dispersion and Foam Stability in Gas Injection Foaming Process. Chin. J. Nonferrous Met. 21 (2), 392-398. doi:10.1016/S1872-5805(11)60064-4

Miyoshi, T., Itoh, M., Akiyama, S., and Kitahara, A. (2000). ALPORAS Aluminum Foam: Production Process, Properties, and Applications. Adv. Eng. Mater. 2 (4), 179-183. doi:10.1002/(sici)1527-2648(200004)2:4<179:: aid-adem $179>3.0 . c 0 ; 2-g$

Song, Z. L., Ma, L. Q., Wu, Z. J., and He, D. P. (2000). Effects of Viscosity on Cellular Structure of Foamed Aluminum in Foaming Process. J. Mater. Sci. 35 (1), 15-20. doi:10.1023/a:1004715926692

Sun, Y. Q., and Gao, T. (2002). The Optimum Wetting Angle for the Stabilization of Liquid-Metal Foams by Ceramic Particles: Experimental Simulations. Metallurgical Mater. Trans. A 33 (10), 3285-3292. doi:10.1007/s11661-0020315-y

Wang, D. Q., and Shi, Z. Y. (2003). Effect of Ceramic Particles on Cell Size and wall Thickness of Aluminum Foam. Mater. Sci. Eng. A 361, 45-49. doi:10.1016/ S0921-5093(03)00557-4

Wang, H., Zhou, X.-y., Long, B., Yang, J., and Liu, H.-z. (2016). Thermal Properties of Closed-Cell Aluminum Foams Prepared by Melt Foaming Technology. Trans. Nonferrous Met. Soc. China 26 (12), 3147-3153. doi:10.1016/s10036326(16)64446-1

Xu, Z. B., and Hao, H. (2014). Electromagnetic Interference Shielding Effectiveness of Aluminum Foams with Different Porosity. J. Alloys Comp. 617, 207-213. doi:10.1016/j.jallcom.2014.07.188

Zhu, H., Sankar, B. V., Haftka, R. T., Venkataraman, S., and Blosser, M. (2004). Optimization of Functionally Graded Metallic Foam Insulation under Transient Heat Transfer Conditions. Struct. Multidisciplinary Optimization 28 (5), 349-355. doi:10.1007/s00158-004-0463-3

Zu, G. Y., Song, B. N., Guan, Z. H., Wang, L., and Yao, G. C. (2011). Preparation of Aluminum Foam Sandwich by Rolling-bonding/Powder Metallurgy Foaming Technology. J. Wuhan Univ. Technology-Materials Sci. Edition 26 (4), 671-674. doi:10.1007/s11595-011-0289-y

Conflict of Interest: The authors declare that the research was conducted in the absence of any commercial or financial relationships that could be construed as a potential conflict of interest.

Publisher's Note: All claims expressed in this article are solely those of the authors and do not necessarily represent those of their affiliated organizations, or those of the publisher, the editors and the reviewers. Any product that may be evaluated in this article, or claim that may be made by its manufacturer, is not guaranteed or endorsed by the publisher.

Copyright (c) 2021 Geng, Li, Li and Huang. This is an open-access article distributed under the terms of the Creative Commons Attribution License (CC BY). The use, distribution or reproduction in other forums is permitted, provided the original author(s) and the copyright owner(s) are credited and that the original publication in this journal is cited, in accordance with accepted academic practice. No use, distribution or reproduction is permitted which does not comply with these terms. 